\title{
SISTEMA DE ANÁLISES EM FLUXO POLIVALENTE PARA A DETERMINAÇÃo ESPECTROFOTOMÉTRICA DE FÁRMACOS
}

\author{
Erlando Schmidt Jr. e Wanessa R. Melchert \\ Instituto de Química, Universidade de São Paulo, CP 26077, 05513-970 São Paulo - SP, Brasil \\ Fábio R. P. Rocha* \\ Centro de Energia Nuclear na Agricultura, Universidade de São Paulo, CP 96, 13400-970 Piracicaba - SP, Brasil
}

Recebido em 17/12/10; aceito em 3/3/11; publicado na web em 5/5/11

\begin{abstract}
A POLIVALENT FLOW SYSTEM FOR THE SPECTROPHOTOMETRIC DETERMINATION OF PHARMACEUTICALS. A flow system based on the sandwich technique is proposed for the sequential determination of ascorbic acid, dipyrone, acetylcysteine, captopril and paracetamol. The procedure is based on the reduction of $\mathrm{Cu}(\mathrm{II})$ by the analytes followed by the spectrophotometric measurement of the complex of $\mathrm{Cu}(\mathrm{I})$ with 2,2'-biquinoline 4,4'-dicarboxylic acid. Linear responses were achieved in the $\mu \mathrm{mol} \mathrm{L^{-1 }}$ range, with coefficients of variation better than $1.7 \%$. Sampling rate was estimated as 60 determinations per hour, consuming $230 \mu \mathrm{g}$ of BQA and generating $2.5 \mathrm{~mL}$ of waste per determination. Results for commercial samples agreed with those obtained by procedures recommended by the American and European pharmacopeias at the $95 \%$ confidence level.
\end{abstract}

Keywords: flow analysis; pharmaceuticals; sequential determination.

\section{INTRODUÇÃO}

Procedimentos para o controle de qualidade de fármacos usualmente utilizam técnicas com equipamentos de custo relativamente alto, como cromatografia a líquido de alta eficiência (HPLC), ou métodos em batelada, que são demorados e consomem quantidades elevadas de reagentes. ${ }^{1,2}$ Como exemplo, pode ser citada a determinação de paracetamol, analgésico amplamente utilizado, cujo procedimento recomendado pela Farmacopeia Europeia emprega a titulação da formulação farmacêutica, após refluxo com $30 \mathrm{~mL}$ de ácido sulfúrico $1,0 \mathrm{~mol} \mathrm{~L} \mathrm{~L}^{-1}$ por $60 \mathrm{~min}$. Este tipo de metodologia não é compatível com os padrões atualmente exigidos no controle de qualidade de preparações farmacêuticas, em relação ao tempo e custo de análises. Por estas razões, o desenvolvimento de procedimentos rápidos, precisos e de baixo custo pode ser muito útil em análises rotineiras.

Sistemas de análises em fluxo têm sido empregados especialmente para mecanizar procedimentos analíticos, minimizar a intervenção do analista, aumentar o número de amostras processadas por unidade de tempo e melhorar a precisão. Estes sistemas também apresentam potencial para o desenvolvimento de procedimentos analíticos mais limpos, com menor produção de resíduos quando comparados aos procedimentos em batelada. ${ }^{3} \mathrm{Um}$ dos inconvenientes para a utilização de sistemas de análises em fluxo em laboratórios de rotina é a necessidade de se empregar diferentes configurações para a determinação de espécies distintas. Esta dificuldade tem sido superada pelo desenvolvimento de sistemas polivalentes, que podem ser aplicados à determinação de várias espécies sem alteração de configuração. ${ }^{4-7}$ Diferentes estratégias têm sido empregadas com esta finalidade, tais como a substituição manual de reagentes ${ }^{4}$ ou de forma mecanizada, utilizando sistemas de análises em fluxo com multicomutação ${ }^{6}$ ou análise por injeção sequencial. ${ }^{7}$ Em muitos casos, as condições experimentais diferem significativamente das ótimas para a determinação

*e-mail: frprocha@cena.usp.br de cada espécie, especialmente quanto à sensibilidade. O potencial da combinação de sistemas de análises em fluxo e quimiometria ${ }^{8}$ e de medidas diretas em fase sólida ${ }^{9,10}$ para determinações simultâneas de espécies de interesse farmacêutico foi recentemente demonstrado.

Neste artigo, é proposto o desenvolvimento de um sistema de análises em fluxo polivalente para a determinação espectrofotométrica sequencial de fármacos. $\mathrm{O}$ procedimento baseia-se na formação do complexo $\mathrm{Cu}$ (I)/BQA, pela redução de $\mathrm{Cu}$ (II) na presença dos analitos (captopril, paracetamol, dipirona, ácido ascórbico e acetilcisteína). A formação deste complexo foi anteriormente explorada para a determinação de cobre, ${ }^{11}$ proteínas, ${ }^{12}$ taninos, ${ }^{13}$ ácido úrico,,${ }^{14}$ polifenóis, ${ }^{15}$ diltiazem ${ }^{16}$ e em estudos de dissolução de fármacos. ${ }^{17}$

\section{PARTE EXPERIMENTAL}

\section{Equipamentos e acessórios}

O módulo de análises foi construído com um injetor proporcional com barra deslizante e tubos de polietileno com 0,7 mm de diâmetro interno. Uma bomba peristáltica (Ismatec, Reglo Digital) equipada com tubos de Tygon ${ }^{\circledR}$ foi utilizada para a propulsão dos fluidos. Um espectrofotômetro UV-Vis (Hitachi, U-3000) equipado com cela de fluxo (Hellma) com caminho óptico de $10 \mathrm{~mm}$ e $80 \mu \mathrm{L}$ de volume interno foi empregado para a medida dos sinais transientes.

Para preparo das amostras, foram utilizados um agitador mecânico (Quimis, Q-225M), uma centrífuga (Quimis, Q-222T108) e um peagâmetro (Metrohm, 654). Um sistema de refluxo foi utilizado para o tratamento de formulações farmacêuticas contendo paracetamol na análise pelo procedimento de referência.

\section{Reagentes e soluções}

Todas as soluções foram preparadas com reagentes de grau analítico e água deionizada. As soluções dos fármacos foram preparadas entre 10 e $400 \mu \mathrm{mol} \mathrm{L}^{-1}$ a partir de diluições em água de soluções 
estoque $1,00 \mathrm{mmol} \mathrm{L}^{-1}$. As soluções de ácido ascórbico e dipirona foram preparadas em meio de $\mathrm{HCl} 0,01 \mathrm{~mol} \mathrm{~L}^{-1}$ para minimizar a oxidação pelo oxigênio dissolvido previamente à análise. As soluções estoque dos fármacos foram padronizadas por iodometria.

Uma solução estoque $0,100 \mathrm{~mol} \mathrm{~L}^{-1}$ de cobre(II) foi preparada por dissolução de $\mathrm{CuSO}_{4} \cdot 5 \mathrm{H}_{2} \mathrm{O}$ em água. Solução $0,2 \mathrm{~mol} \mathrm{~L}^{-1}$ de acetato de amônio foi preparada por dissolução de $\mathrm{NH}_{4} \mathrm{CH}_{3} \mathrm{CO}_{2}$ em água, sendo o $\mathrm{pH}$ ajustado para 7,0. $\mathrm{O}$ reagente $\mathrm{R}_{1}$ foi preparado a partir destas soluções, resultando em 2,00 $\mathrm{mmol} \mathrm{L}^{-1}$ de $\mathrm{Cu}(\mathrm{II})$ em $0,1 \mathrm{~mol}$ $\mathrm{L}^{-1}$ de acetato de amônio.

A solução de BQA 6,00 mmol L-1 $\left(\mathrm{R}_{2}\right)$ foi preparada a partir da dissolução de $116 \mathrm{mg}$ do sal dissódico do ácido 4,4'-dicarboxi2,2'-biquinolínico em $50 \mathrm{~mL}$ de água. A solução transportadora foi hidróxido de amônio 7,5 mmol L-1, preparada pela diluição de 520 $\mu \mathrm{L}$ de $\mathrm{NH}_{4} \mathrm{OH}$ concentrado em $500 \mathrm{~mL}$ de água.

Para a avaliação do efeito dos excipientes foram preparadas soluções/suspensões dos analitos $\left(50 \mu \mathrm{mol} \mathrm{L}^{-1}\right)$ na presença de cada espécie empregada em preparações comerciais (lactose, glicose, sacarose, polietilenoglicol, metabissulfito de sódio, cafeína, sacarina, sorbitol, ácido cítrico, bicarbonato de sódio, carbonato de sódio, celulose microcristalina, estearato de magnésio, ácido esteárico, amido, dióxido de silício e dióxido de titânio). Os excipientes testados foram selecionados a partir da composição das formulações farmacêuticas descritas nas bulas. As soluções foram analisadas logo após o preparo, enquanto as suspensões das espécies insolúveis foram submetidas à agitação por 10 min, seguida de centrifugação por 5 min e filtração.

\section{Preparo de amostras}

As amostras foram adquiridas em drogarias locais. Para a análise, de 10 a 20 comprimidos de cada medicamento foram pesados e finamente pulverizados em almofariz. Uma massa adequada de cada amostra (ca. $200 \mathrm{mg}$ ) foi agitada ( $c a .10 \mathrm{~min}$ ) com água deionizada ou solução de $\mathrm{HCl} 0,01 \mathrm{~mol} \mathrm{~L}^{-1}$ para a extração dos analitos. A mistura resultante foi centrifugada e filtrada. Previamente às medidas analíticas, as amostras foram diluídas a concentrações intermediárias no intervalo de resposta dos procedimentos (entre 60 e $140 \mu \mathrm{mol} \mathrm{L}^{-1}$ ), considerando os valores rotulados.

\section{Módulo de análises e procedimento}

O diagrama de fluxos do módulo de análises proposto é apresentado na Figura 1. Na posição ilustrada, a amostra (A) e os reagentes $\left(R_{1}\right.$ e $\left.R_{2}\right)$ foram aspirados simultaneamente para preencher as alças de amostragem $\left(\mathrm{L}_{1}-\mathrm{L}_{3}\right)$. Na posição alternativa do injetor, as soluções foram simultaneamente inseridas na solução transportadora e a mis-

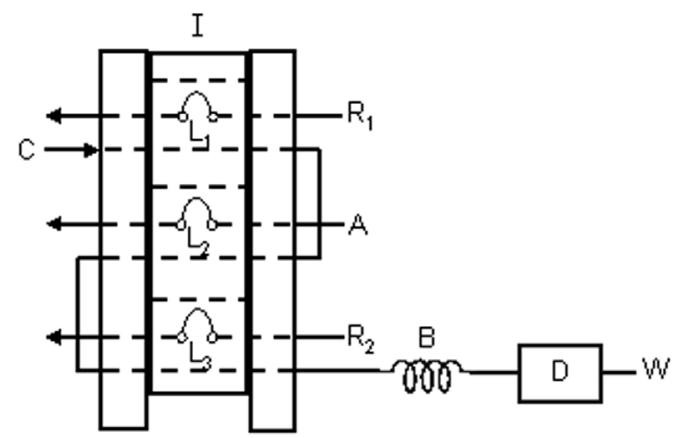

Figura 1. Diagrama do sistema de análises em fluxo. I: injetor proporcional; A: amostra; $R_{l}: C u^{2+}$ em solução tampão; $R_{2}: B Q A ; C$ : transportador; $B$ : reator helicoidal; $L_{1}, L_{2}$ e $L_{3}$ : alças de amostragem; D: espectrofotômetro e W: descarte tura ocorreu por dispersão mútua nas interfaces. A zona de amostra foi transportada através do reator helicoidal de $100 \mathrm{~cm}$, em direção à cela de fluxo para a medida espectrofotométrica em $558 \mathrm{~nm}$. As medidas foram baseadas em altura de pico e realizadas em triplicata.

Procedimentos descritos nas Farmacopeias Americana ${ }^{1}$ e Europeia $^{2}$ foram utilizados como referência para comparação dos resultados das análises dos fármacos, exceto dipirona, que foi analisado por um procedimento em fluxo baseado em iodometria. ${ }^{18}$ As amostras de ácido ascórbico, acetilcisteína e captopril foram analisadas por titulação iodométrica, como descrito na Farmacopeia Americana ${ }^{1}$. Amostras de paracetamol foram tituladas com sulfato de cério(IV), como descrito na Farmacopeia Europeia. ${ }^{2}$ Todas as medidas pelos procedimentos de referência também foram realizadas em triplicata.

\section{RESULTADOS E DISCUSSÃO}

\section{Aspectos gerais}

Com o objetivo de facilitar o emprego em análises de rotina, foi proposto um módulo de análises com configuração simples e robusta, além de explorar um mesmo método para a determinação dos diferentes analitos. O sistema de análises em fluxo foi projetado com configuração em linha única, utilizando um injetor proporcional para a inserção simultânea de amostras e reagentes. A estratégia de amostragem adotada foi intercalar a alíquota de amostra entre as alíquotas dos reagentes (técnica sanduíche). ${ }^{19}$ Desta forma, a interação entre o íon metálico e o ligante ocorreu após interação do íon metálico com o analito redutor, permitindo a formação do complexo com cobre(I). Esta configuração foi também selecionada para minimizar o consumo de reagentes e a geração de resíduos.

A determinação espectrofotométrica foi baseada na geração do complexo violeta de $\mathrm{Cu}(\mathrm{I})$ com o ânion do ácido 4,4'-dicarboxi2,2'-biquinolínico (BQA). As estruturas dos fármacos estudados estão apresentadas na Figura 2. Estas espécies reduziram cobre(II) (Equação 1) e a espécie reduzida formou o complexo com BQA (Equação 2), com máximos de absorção em 354 e 558 nm e absortividades molares ${ }^{11}$ de $4,6 \times 10^{4} \mathrm{e} 7,7 \times 10^{3} \mathrm{~L} \mathrm{~mol}^{-1} \mathrm{~cm}^{-1}$, respectivamente. Apesar da menor absortividade, o comprimento de onda no visível foi selecionado para as medidas espectrofotométricas devido à absorção de radiação pelos analitos e acetato na região ultravioleta. A estequiometria do complexo é de 1:2 como mostrado na Equação 2.

$$
\begin{aligned}
& \mathrm{x} \mathrm{Cu}^{2+}+\mathrm{y} \text { analito }_{\text {(reduzido) }} \rightarrow \mathrm{x} \mathrm{Cu}^{+}+\mathrm{y}_{\text {analito }_{\text {(oxidado) }}} \\
& \mathrm{Cu}^{+}+2 \mathrm{BQA}^{2-} \rightarrow\left[\mathrm{Cu}(\mathrm{BQA})_{2}\right]^{3-}
\end{aligned}
$$

a<smiles>CC(=O)N[C@@H](CS)C(=O)O</smiles><smiles>CC(CS)C(=O)N1CCCC1C(=O)O</smiles>

d<smiles>Cc1c(N(C)CS(=O)(=O)O)c(=O)n(-c2ccccc2)n1C</smiles><smiles>CC(=O)Nc1ccc(O)cc1</smiles>

Figura 2. Estruturas moleculares dos fármacos estudados: a-acetilcisteína; $b$-ácido ascórbico; $c$-captopril; d-dipirona; $e$-paracetamol

\section{Otimização das variáveis}

Os parâmetros químicos (concentração dos reagentes, $\mathrm{pH}$ da solução tampão e composição da solução transportadora) e físicos 
(volumes de amostra e de reagentes; tamanho do reator helicoidal e vazão) foram otimizados pelo método univariado. Na otimização foram consideradas a magnitude do sinal analítico, a precisão e o consumo de reagentes. As faixas estudadas e valores selecionados para cada parâmetro estão listados na Tabela 1

Tabela 1. Parâmetros otimizados para a determinação sequencial de fármacos redutores

\begin{tabular}{lcc}
\hline Parâmetro & Faixa estudada & Valor selecionado \\
\hline Volume de $\mathrm{R}_{1}(\mu \mathrm{L})$ & $50-250$ & 100 \\
Volume de amostra $(\mu \mathrm{L})$ & $25-250$ & 150 \\
Volume de $\mathrm{R}_{2}(\mu \mathrm{L})$ & $50-250$ & 100 \\
Comprimento do reator $(\mathrm{cm})$ & $50-200$ & 100 \\
Vazão do transportador $\left(\mathrm{mL} \mathrm{min}^{-1}\right)$ & $1,5-3,5$ & 2,5 \\
Concentração de $\mathrm{Cu}(\mathrm{II})\left(\mathrm{mmol} \mathrm{L}^{-1}\right)$ & $1,00-8,00$ & 2,00 \\
Concentração de $\mathrm{BQA}\left(\mathrm{mmol} \mathrm{L}^{-1}\right)$ & $3,00-12,0$ & 6,00 \\
Concentração de $\mathrm{NH}_{4} \mathrm{OH}\left(\mathrm{mmol} \mathrm{L}^{-1}\right)$ & $7,5-30$ & 7,5 \\
Concentração de $\mathrm{NH}_{4} \mathrm{CH}_{3} \mathrm{CO}_{2}\left(\mathrm{~mol} \mathrm{~L}^{-1}\right)$ & $0,01-1,0$ & 0,1 \\
pH da solução de $\mathrm{NH}_{4} \mathrm{CH}_{3} \mathrm{CO}_{2}$ & $6,0-8,0$ & 7,0 \\
\hline
\end{tabular}

O efeito da concentração de $\mathrm{Cu}$ (II) na solução $\mathrm{R}_{1}$ foi avaliado entre 1,0 e $8,0 \mathrm{mmol} \mathrm{L}^{-1}$ usando soluções $50 \mu \mathrm{mol} \mathrm{L}^{-1}$ de cada analito. Neste estudo foi utilizada uma solução de BQA $6,00 \mathrm{mmol} \mathrm{L}^{-1}\left(\mathrm{R}_{2}\right)$, água como transportador, $\mathrm{NH}_{4} \mathrm{CH}_{3} \mathrm{CO}_{2} 0,1 \mathrm{~mol} \mathrm{~L}^{-1} \mathrm{em} \mathrm{R}_{1}$ e reator helicoidal de $100 \mathrm{~cm}$. Os resultados estão apresentados na Figura 3. Em cada condição, as medidas foram corrigidas considerando o branco analítico. Maiores sinais analíticos foram geralmente observados com maiores concentrações de $\mathrm{Cu}(\mathrm{II})$, porém também foi observado aumento do branco analítico. Por exemplo, quando uma solução de $\mathrm{Cu}$ (II) 4,00 mmol L ${ }^{-1}$ foi utilizada para a determinação de acetilcisteína, o sinal analítico foi 1,7 vezes superior ao obtido com uma solução 2,00 mmol $\mathrm{L}^{-1}$; entretanto, o sinal do branco analítico foi $c a$. 20 vezes maior. Isto se deve à precipitação do excesso de cobre(II) com BQA, o que causa espalhamento de radiação, principalmente em concentrações de $\mathrm{Cu}$ (II) superiores a 2,00 $\mathrm{mmol} \mathrm{L}^{-1}$. Considerando a magnitude dos sinais analíticos e do branco, a concentração de $2,00 \mathrm{mmol} \mathrm{L}^{-1} \mathrm{de} \mathrm{Cu}$ (II) foi selecionada. Nestas condições, há um excesso de $\mathrm{Cu}$ (II) de pelo menos 10 vezes em relação à maior concentração de analito avaliada.

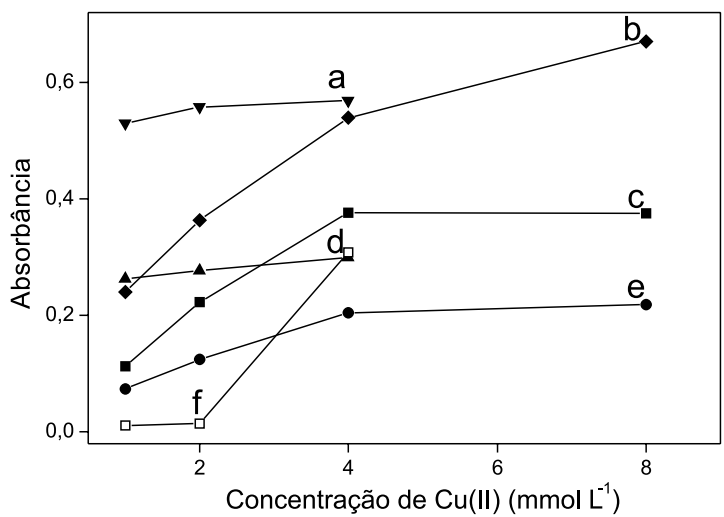

Figura 3. Efeito da concentração de Cu(II) sobre o sinal analítico: a -dipirona; $b$-paracetamol; c-acetilcisteína; $d$-captopril; $e$-ácido ascórbico. $f$-corresponde ao branco analítico

A concentração de BQA foi avaliada entre 3,00 e 12,0 mmol $\mathrm{L}^{-1}$ para os diferentes fármacos, usando uma solução $2,00 \mathrm{mmol} \mathrm{L}^{-1}$
$\mathrm{Cu}$ (II) em $\mathrm{R}_{1}$ e as condições experimentais citadas anteriormente. $\mathrm{Na}$ Figura 4, observa-se que os sinais analíticos e de branco não foram alterados significativamente com a concentração de BQA (exceto para o paracetamol). Desta forma, com o objetivo de diminuir o consumo do reagente complexante, a concentração $6,00 \mathrm{mmol} \mathrm{L}^{-1}$ de BQA foi selecionada para estudos posteriores.

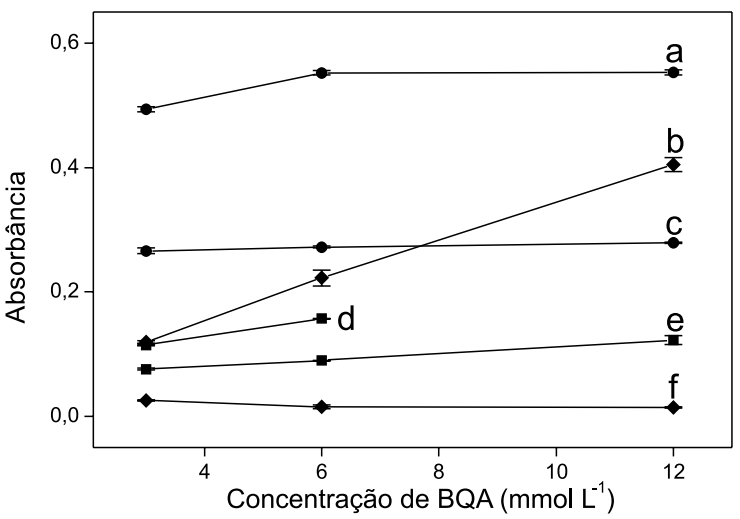

Figura 4. Efeito da concentração de BQA sobre o sinal analítico: a - dipiro$n a ; b$ - paracetamol; $c$-captopril; $d$-acetilcisteína; $e$ - ácido ascórbico; $f$-branco analítico

Os efeitos dos volumes de reagentes e amostra foram avaliados entre 50 e $250 \mu \mathrm{L}$ (alças de amostragem entre 10 e $50 \mathrm{~cm}$ ), resultando em pequena influência sobre o sinal analítico, devido ao excesso dos reagentes mesmo para os menores volumes avaliados. Alças de amostragem de $20 \mathrm{~cm}(100 \mu \mathrm{L})$ foram selecionadas por proporcionar a melhor razão entre sinal analítico e do branco. Para a alça de amostra, a melhor resposta foi atingida com um comprimento de $30 \mathrm{~cm}(150 \mu \mathrm{L})$. Maiores volumes resultaram na formação de picos duplos, devido à mistura deficiente entre os reagentes, que ocorre exclusivamente por dispersão.

O efeito da vazão do transportador foi avaliado entre 1,5 e 3,5 $\mathrm{mL}$ min $^{-1}$. Como este parâmetro altera o tempo de residência e a dispersão da zona de amostra, a resposta analítica foi distinta para cada analito. Por exemplo, uma vazão de $1,5 \mathrm{~mL} \mathrm{~min}^{-1}$ resultou em maior resposta para ácido ascórbico, menor para acetilcisteína e na formação de picos duplos nas medidas de paracetamol. Usando uma vazão de $3,5 \mathrm{~mL} \mathrm{~min}^{-1}$, a reprodutibilidade diminuiu e os sinais analíticos foram menores para a maioria dos analitos. Tendo em vista que vazões baixas prejudicam a frequência de amostragem, a vazão de $2,5 \mathrm{~mL} \mathrm{~min}^{-1}$ foi selecionada como condição de compromisso.

Usando somente água deionizada como transportador, foi observada deriva de linha base, causada pelo depósito do sólido verde de $\mathrm{Cu}(\mathrm{BQA})$ na cela de fluxo. Hidróxido de amônio foi adicionado ao transportador para melhorar a limpeza da cela de fluxo, ${ }^{13}$ evitando a precipitação de $\mathrm{Cu}$ (II) pela formação do complexo estável ${ }^{20}$ $\left[\mathrm{Cu}\left(\mathrm{NH}_{3}\right)_{4}\right]^{2+}, \mathrm{K}_{\mathrm{f}}=1,07 \times 10^{12}$. Soluções com concentrações de $\mathrm{NH}_{4} \mathrm{OH}$ superiores a 7,5 mmol L-1 resultaram em respostas menores, devido à diminuição da concentração de cobre(II) livre em excesso de amônia. Portanto, a concentração de $7,5 \mathrm{mmol} \mathrm{L}^{-1}$ foi selecionada por apresentar melhores sinais analíticos e limpeza adequada da cela de fluxo. Nestas condições, a deriva de linha base foi desprezível, mesmo após 6 h de operação contínua.

O efeito da concentração de tampão foi investigado entre 0,01 e $1,00 \mathrm{~mol} \mathrm{~L}^{-1} \mathrm{e}$ os resultados obtidos foram praticamente equivalentes. Entretanto, um aumento de linha base foi observado quando uma solução $0,01 \mathrm{~mol} \mathrm{~L}^{-1}$ foi utilizada, devido à formação do precipitado $\mathrm{Cu}$ (BQA), o que justifica a escolha da solução $0,1 \mathrm{~mol} \mathrm{~L}^{-1}$. A formação de precipitado na zona de amostra é evitada pela complexação 
de $\mathrm{Cu}(\mathrm{II})$ por $\operatorname{acetato}^{20}\left(\mathrm{~K}_{\mathrm{f}}=2,0 \times 10^{3}\right)$ e amônia. Embora este ligante esteja presente em baixa concentração em solução de acetato de amônio em pH 7,0, a elevada constante de formação favorece a dissolução do sólido CuBQA. O efeito da acidez da solução tampão foi avaliado entre pH 6,0 e 8,0. Apesar de resultados distintos terem sido obtidos para cada fármaco, a melhor resposta, considerando o sinal analítico e a reprodutibilidade, foi obtida em $\mathrm{pH}$ 7,0, no qual o sistema tampão apresenta sua maior capacidade tamponante. Este $\mathrm{pH}$ coincide com o empregado em estudos anteriores, ${ }^{13-17}$ exceto no caso da determinação de proteínas. ${ }^{12}$

O comprimento do reator afeta tanto o tempo de residência, quanto a dispersão da amostra. Este parâmetro foi avaliado entre 50 e $150 \mathrm{~cm}$. Para a determinação de acetilcisteína, ácido ascórbico e paracetamol, o reator de $100 \mathrm{~cm}$ apresentou sinais analíticos maiores, já para determinação de captopril e dipirona, o reator de $50 \mathrm{~cm}$ apresentou melhores respostas. Este resultado indica que a redução de $\mathrm{Cu}$ (II) por estas espécies deve ser mais rápida e o aumento do tempo de residência não compensa o aumento da dispersão da amostra. Considerando a sensibilidade e reprodutibilidade para os diferentes analitos, foi selecionado um reator helicoidal de $100 \mathrm{~cm}$.

\section{Estabilidade das soluções}

Devido à determinação de espécies com características redutoras, o efeito da presença de oxigênio dissolvido e do tempo de armazenamento das soluções de referência foi avaliado. Todas as soluções foram preparadas por diluições em água, imediatamente antes das medidas analíticas. Este procedimento gerou bons resultados, exceto para dipirona e ácido ascórbico. Visando aumentar a estabilidade, soluções de referência destes fármacos foram preparadas em meio ácido.

Na ausência de ácido nas soluções de referência, o sinal analítico de dipirona aumentava gradativamente com o tempo. Golubitskii et $a l .{ }^{21}$ demonstraram que a decomposição de dipirona em soluções aquosas envolve três etapas reacionais, sendo a primeira mais rápida, catalisada por ácido e que produz sulfito como sub-produto. Sulfito é um conhecido agente redutor, que aumenta a concentração de $\mathrm{Cu}(\mathrm{I})$ e, portanto, o sinal analítico. Um estudo de estabilidade da solução de dipirona foi realizado em soluções com $\mathrm{pH}$ variando entre 2,0 e 5,6 e foi observada maior estabilidade da solução em $\mathrm{pH}$ abaixo de 3,0. Em pH 2,0, o sinal analítico foi aproximadamente 50\% superior ao sinal obtido com a solução em pH 3,0 (Figura 5). Desta forma, as soluções de referência de dipirona e os extratos obtidos das amostras foram preparados nesta acidez.

Soluções de ácido ascórbico em meio neutro ou alcalino são rapidamente oxidadas pelo oxigênio dissolvido, pois o ânion ascorbato apresenta caráter fortemente redutor. ${ }^{22}$ Como a velocidade de oxidação por oxigênio é menor em soluções ácidas, as soluções de ácido ascórbico foram preparadas em meio de $\mathrm{HCl} 0,01 \mathrm{~mol} \mathrm{~L}^{-1}$.

\section{Características analíticas}

Nas condições otimizadas, empregando o diagrama de fluxos

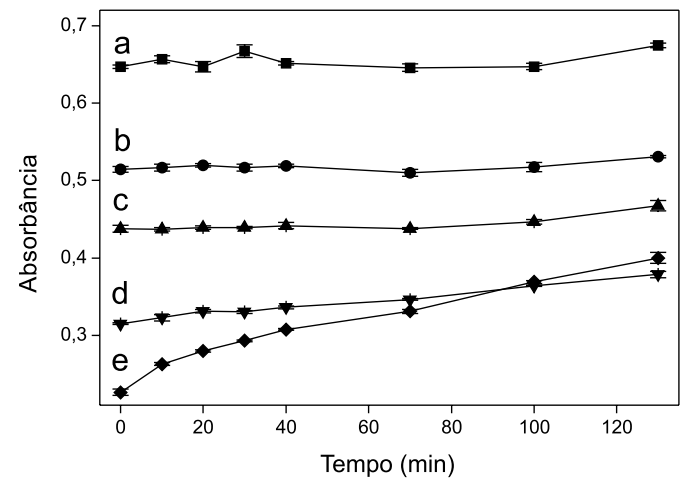

Figura5. EfeitodopHnaestabilidade de solução de dipirona. $a-p H 2,0 ; b-p H 2,5$; $c-p H 3,0 ; d-p H 4,0 ; e-p H 5,6$

apresentado na Figura 1, foram construídas curvas de calibração, estimados os limites de detecção e os coeficientes de variação para os diferentes fármacos. $\mathrm{O}$ limite de detecção foi estimado de acordo com as recomendações da IUPAC, considerando três vezes o desvio das medidas realizadas com o branco analítico (99,7\% de confiança). Essas características analíticas estão listadas na Tabela 2. A frequência de amostragem foi estimada em 60 determinações/h, com consumo de $230 \mu \mathrm{g}$ de BQA e geração de 2,5 mL de resíduo por determinação. $\mathrm{O}$ procedimento pode ser considerado ambientalmente amigável por minimizar a quantidade de resíduos gerados. Além disso, o reagente mais tóxico (BQA) pode ser recuperado utilizando um procedimento descrito anteriormente. ${ }^{16}$

\section{Efeito dos excipientes}

Os efeitos das espécies normalmente presentes em formulações farmacêuticas foram avaliados. As quantidades máximas toleradas (interferência menor que 5\%) de cada excipiente estão apresentadas na Tabela 3. Os resultados indicam que é possível a determinação dos analitos sem a ocorrência de interferências, visto que os excipientes são tolerados em quantidades superiores às usualmente encontradas nas formulações comerciais.

\section{Análises de formulações farmacêuticas}

As amostras foram preparadas conforme anteriormente descrito e analisadas pelo procedimento proposto e por procedimentos de referência descritos na literatura. ${ }^{1,2,18}$ Os resultados estão apresentados nas Tabelas 4-8. Os resultados obtidos para as amostras dos diferentes fármacos utilizando o método proposto não apresentaram diferenças significativas em nível de confiança de $95 \%$ quando comparados aos obtidos com os métodos de referência.

Na determinação de paracetamol, a amostra 4 não pôde ser quantificada pelo método de referência, pois a formulação farmacêutica continha ácido acetilsalicílico. O produto da degradação gerado durante a etapa de refluxo (ácido salicílico) forma complexo com

Tabela 2. Características analíticas dos procedimentos para os diferentes fármacos

\begin{tabular}{lcccc}
\hline & Resposta linear $\left.(\mu \mathrm{mol} \mathrm{L})^{-1}\right)$ & Limite de detecção $\left(\mu \mathrm{mol} \mathrm{L}^{-1}\right)$ & Sensibilidade $\left(\mathrm{L} \mathrm{mol}{ }^{-1}\right)$ & Coeficiente de variação $(\%)(\mathrm{n}=20)$ \\
\hline Acetilcisteína & $10-400$ & 1,5 & 2793 & 1,7 \\
Ácido ascórbico & $10-200$ & 2,8 & 3428 & 1,3 \\
Captopril & $10-400$ & 3,0 & 2582 & 0,5 \\
Dipirona & $20-140$ & 2,1 & 3494 & 1,4 \\
Paracetamol & $10-100$ & 3,1 & 4134 & 1,7 \\
\hline
\end{tabular}


Tabela 3. Concentrações $\left(\mathrm{g} \mathrm{L}^{-1}\right)$ máximas toleradas (interferência menor que 5\%) de excipientes na determinação dos fármacos

\begin{tabular}{|c|c|c|c|c|c|}
\hline Excipiente & Acetilcisteína & Ácido ascórbico & Captopril & Dipirona & Paracetamol \\
\hline Ácido cítrico & 0,02 & 0,02 & - & - & - \\
\hline Ácido esteárico & - & 2,0 & - & - & 2,0 \\
\hline Amido & - & 2,0 & - & 2,0 & 2,0 \\
\hline Bicarbonato de sódio & 1,0 & 0,1 & - & - & - \\
\hline Cafeína & - & - & - & 2,0 & - \\
\hline Carbonato de sódio & - & 0,02 & - & - & - \\
\hline Celulose microcristalina & - & 2,0 & 2,0 & 2,0 & 2,0 \\
\hline Dióxido de silício & - & - & 1,5 & 2,0 & - \\
\hline Dióxido de titânio & - & - & - & 2,0 & - \\
\hline Estearato de magnésio & - & 2,0 & - & 2,0 & 2,0 \\
\hline Fosfato de sódio monobásico & 2,0 & - & - & - & - \\
\hline Glicose & - & - & 2,0 & - & - \\
\hline Lactose & - & - & 2,0 & 2,0 & - \\
\hline Metabissulfito de sódio & - & - & - & - & 0,005 \\
\hline Polietilenoglicol & - & 1,0 & - & 2,0 & 2,0 \\
\hline Sacarina & 2,0 & 2,0 & - & - & - \\
\hline Sacarose & 2,0 & 2,0 & 2,0 & 2,0 & - \\
\hline Sorbitol & - & 2,0 & - & - & - \\
\hline
\end{tabular}

Tabela 4. Determinação de ácido ascórbico em formulações farmacêuticas

\begin{tabular}{|c|c|c|c|}
\hline \multirow[b]{2}{*}{ Amostra } & \multicolumn{3}{|c|}{ Quantidade de ácido ascórbico (mg/comprimido) } \\
\hline & $\begin{array}{l}\text { Valor } \\
\text { rotulado }\end{array}$ & $\begin{array}{c}\text { Método } \\
\text { proposto* }\end{array}$ & $\begin{array}{l}\text { Método de } \\
\text { referência }{ }^{1, *}\end{array}$ \\
\hline 1 & 500 & $491 \pm 2$ & $500 \pm 5$ \\
\hline 2 & 500 & $461 \pm 6$ & $528 \pm 5$ \\
\hline 3 & 1000 & $987 \pm 6$ & $1021 \pm 14$ \\
\hline 4 & 1000 & $951 \pm 12$ & $964 \pm 11$ \\
\hline 5 & 200 & $229 \pm 1$ & $204 \pm 3$ \\
\hline
\end{tabular}

*Médias e desvios para 3 replicatas

Tabela 5. Determinação de captopril em formulações farmacêuticas

\begin{tabular}{|c|c|c|c|}
\hline \multirow[b]{2}{*}{ Amostra } & \multicolumn{3}{|c|}{ Quantidade de captopril (mg/comprimido) } \\
\hline & $\begin{array}{l}\text { Valor } \\
\text { rotulado }\end{array}$ & $\begin{array}{l}\text { Método } \\
\text { proposto* }\end{array}$ & $\begin{array}{l}\text { Método de } \\
\text { referência }{ }^{1, *}\end{array}$ \\
\hline 1 & 12,5 & $12,0 \pm 0,2$ & $12,1 \pm 0,1$ \\
\hline 2 & 12,5 & $11,7 \pm 0,2$ & $11,7 \pm 0,1$ \\
\hline 3 & 12,5 & $12,4 \pm 0,2$ & $12,6 \pm 0,1$ \\
\hline 4 & 50 & $49,0 \pm 0,7$ & $48,9 \pm 0,7$ \\
\hline 5 & 25 & $23,9 \pm 0,4$ & $23,6 \pm 0,2$ \\
\hline
\end{tabular}

*Médias e desvios para 3 replicatas

Tabela 6. Determinação de dipirona em formulações farmacêuticas

\begin{tabular}{lccc}
\hline Amostra & \multicolumn{3}{c}{ Quantidade de dipirona (mg/comprimido) } \\
Valor & $\begin{array}{c}\text { Método } \\
\text { proposto* }\end{array}$ & $\begin{array}{c}\text { Método de } \\
\text { referência }\end{array}$ \\
\hline 1 & 500 & $563 \pm 7$ & $539 \pm 12$ \\
2 & 500 & $556 \pm 3$ & $470 \pm 21$ \\
3 & 300 & $316 \pm 5$ & $318 \pm 7$ \\
4 & 500 & $560 \pm 2$ & $535 \pm 2$ \\
5 & 500 & $513 \pm 7$ & $562 \pm 9$ \\
6 & 250 & $259 \pm 3$ & $226 \pm 17$ \\
7 & 250 & $256 \pm 3$ & $220 \pm 9$ \\
8 & 500 & $520 \pm 5$ & $474 \pm 16$ \\
\hline
\end{tabular}

*Médias e desvios para 3 replicatas
Tabela 7. Determinação de acetilcisteína em formulações farmacêuticas

\begin{tabular}{|c|c|c|c|}
\hline \multirow[b]{2}{*}{ Amostra } & \multicolumn{3}{|c|}{ Quantidade de acetilcisteína (mg/comprimido) } \\
\hline & $\begin{array}{l}\text { Valor } \\
\text { rotulado }\end{array}$ & $\begin{array}{c}\text { Método } \\
\text { proposto* }\end{array}$ & $\begin{array}{l}\text { Método de } \\
\text { referência }\end{array}$ \\
\hline 1 & 100 & $232 \pm 2$ & $233 \pm 1$ \\
\hline 2 & 100 & $112 \pm 1$ & $115 \pm 1$ \\
\hline 3 & 20 & $20,4 \pm 0,3$ & $20,5 \pm 0,6$ \\
\hline 4 & 20 & $18,0 \pm 0,1$ & $18,2 \pm 0,3$ \\
\hline
\end{tabular}

*Médias e desvios para 3 replicatas

Tabela 8. Determinação de paracetamol em formulações farmacêuticas

\begin{tabular}{|c|c|c|c|}
\hline \multirow[b]{2}{*}{ Amostra } & \multicolumn{3}{|c|}{ Quantidade de paracetamol (mg/comprimido) } \\
\hline & $\begin{array}{c}\text { Valor } \\
\text { rotulado }\end{array}$ & $\begin{array}{c}\text { Método } \\
\text { proposto* }\end{array}$ & $\begin{array}{c}\text { Método de } \\
\text { referência }{ }^{2, *}\end{array}$ \\
\hline 1 & 750 & $734 \pm 8$ & $744 \pm 13$ \\
\hline 2 & 500 & $479 \pm 5$ & $470 \pm 6$ \\
\hline 3 & 750 & $754 \pm 12$ & $769 \pm 5$ \\
\hline 4 & 150 & $154 \pm 1$ & - \\
\hline 5 & 750 & $755 \pm 3$ & $725 \pm 13$ \\
\hline
\end{tabular}

*Médias e desvios para 3 replicatas

o $\mathrm{Fe}^{3+}$ proveniente do indicador ferroína utilizado na titulação com sulfato de cério(IV). Este complexo possui coloração alaranjada, o que dificulta a visualização do ponto final da titulação. Esta limitação não foi observada para o procedimento proposto.

\section{CONCLUSÕES}

O procedimento proposto é uma alternativa vantajosa aos métodos oficiais para laboratórios de controle de qualidade, visto que minimiza o tempo de análise, a quantidade de reagentes e, consequentemente, o custo operacional e a geração de resíduos. É um procedimento de fácil aplicação, já que a mesma configuração de módulo de análises é utilizada para a determinação de diversos analitos, e ambientalmente mais amigável, por não empregar reagentes tóxicos e gerar pequena 
quantidade de efluentes. O estudo de interferentes e a comparação com procedimentos de referência demonstraram que o procedimento proposto é adequado para análise de formulações farmacêuticas comerciais.

\section{AGRADECIMENTOS}

As bolsas e auxílios concedidos pelo CNPq e FAPESP.

\section{REFERÊNCIAS}

1. United States Pharmacopeia; United States Pharmacopeial Convention, $30^{\text {rd }}$ ed., 2007.

2. European Pharmacopoeia; European Directorate for the Quality of Medicines, $5^{\text {th }}$ ed., 2005.

3. Melchert, W. R.; Infante, C. M. C.; Rocha, F. R. P.; Microchem. J. 2007, $85,209$.

4. Silva, F. V.; Nogueira, A. R. A.; Souza, G. B.; Zagatto, E. A. G.; Anal. Chim. Acta 1998, 370, 39.

5. Medina, A. R.; Córdova, M. L. F.; Molina-Diaz, A.; J. Pharm. Biomed. Anal. 1999, 21, 983.

6. Rocha, F. R. P.; Fatibello-Filho, O.; Reis, B. F.; Talanta 2003, 59, 191.

7. Pimenta, A. M.; Montenegro, M. C. B. S. M.; Araújo, A. N.; Calatayud, J. M.; J. Pharm. Biomed. Anal. 2006, 40, 16.

8. Saurina, J.; Trends Anal. Chem. 2010, 29, 1027.
9. Ruiz-Medina, A.; Llorent-Martínez, E. J.; J. Pharm. Biomed. Anal. 2010, $53,250$.

10. Rocha, F. R. P.; Raimundo, I. M.; Teixeira, L. S. G.; Anal. Lett. 2011, 44, 1.

11. Brenner, A. J.; Harris, E. D.; Anal. Biochem. 1995, 226, 80.

12. Smith, P. K.; Krohn, R. I.; Hermanson, G. T.; Mallia, A. K.; Gartner, F. H.; Provenzano, M. D.; Fujimoto, E. K.; Goeke, N. M.; Olson, B. J.; Klenk, D. C.; Anal. Biochem. 1985, 150, 76.

13. Moya, H. D.; Dantoni, P.; Rocha, F. R. P.; Coichev, N.; Microchem. J. 2008, $88,21$.

14. Rocha, D. L.; Rocha, F. R. P.; Microchem. J. 2010, 94, 53.

15. Marino, D. C.; Sabino, L. Z. L.; Armando Jr., J.; Ruggiero, A. A.; Moya, H. D.; J. Agric. Food Chem. 2009, 57, 11061.

16. Sabino, L. Z. L.; Marino, D. C.; Moya, H. D.; Can. J. Chem. 2010, 88, 533.

17. Sanchez, M. A.; Rocha, D. L.; Melchert, W. R.; Rocha, F. R. P.; J. Braz. Chem. Soc., no prelo.

18. Fatibello-Filho, O.; Pereira, A. V.; Penckowski, L.; Vosgerau, M.; Sassá, M. F.; Quim. Nova 2002, 25, 553.

19. Alonso, J.; Bartrolí, J.; Del Valle, M.; Escalada, M.; Barber, R.; Anal. Chim. Acta 1987, 199, 191.

20. Lurie, J.; Handbook of Analytical Chemistry, Mir Publishers: Moscou, 1978.

21. Golubitskii, G. B.; Kostarnoi, A. V.; Budko, E. V.; Ivanov, V. M.; Basova, E. M.; J. Anal. Chem. 2006, 61, 997.

22. Mehrotra, U. S.; Agrawal, M. C.; Mushran, S. P.; J. Phys. Chem. 1969, 73, 1996. 